\title{
Estratégias fisioterapêuticas para alívio da dor durante trabalho de parto
}

O parto humanizado é preconizado pela Organização Mundial da Saúde (OMS) para garantir saúde materna e neonatal, reduzir as taxas de mortalidades nessa população e restringir o uso de recursos tecnológicos. O fisioterapeuta é o profissional habilitado para acompanhar a parturiente em trabalho de parto, garantindo seu protagonismo e ofertando recursos e técnicas não farmacológicas para a promoção de analgesia, segurança e conforto. 0 objetivo deste estudo é realizar uma revisão bibliográfica para identificar as principais estratégias utilizadas na assistência fisioterapêutica durante o trabalho de parto e parto humanizado para alívio da dor. Para isso, realizou-se uma revisão bibliográfica, nos últimos 10 anos, entre novembro de 2017 e abril de 2018, nas bases de dados PubMed, Google Acadêmico, Periódicos Capes, LILACS e SCIELO, por ensaios clínicos randomizados, revisões bibliográficas e metanálises de efeitos randômicos, além de manuais oficiais do Ministério da Saúde. A seleção de artigos elegíveis e a avaliação de sua qualidade metodológica foram feitas independentemente por dois revisores, e as palavras-chave utilizadas foram: 'fisioterapia'; 'parto humanizado'; 'dor de parto'; e 'trabalho de parto', e seus correlatos na língua inglesa: 'physical therapy'; 'humanized labor'; 'labor pain'; e 'labor'. As estratégias fisioterapêuticas utilizadas durante o trabalho de parto e parto humanizado para alívio da dor foram desde técnicas ocidentais a orientais, tais quais: posicionamento e deambulação bola suíça, percepção respiratória, relaxamento, TENS, massagem, banhos quentes, crioterapia, reflexologia, auriculoterapia, acupuntura e acupressão.

Palavras-chave: Fisioterapia; Parto humanizado; Dor de parto; Trabalho de parto.

\section{Physiotherapeutic strategies for pain relief during labor}

Humanized childbirth is advocated by the World Health Organization (WHO) to ensure maternal and newborn health, reduce mortality rates in this population and restrict the use of technological resources. The physiotherapist is the professional qualified to accompany the parturient in labor, guaranteeing her protagonism and offering resources and non-pharmacological techniques for the promotion of analgesia, safety and comfort. The objective of this study is to perform a bibliographic review to identify the main strategies used in physiotherapeutic assistance during labor and humanized labor for pain relief. For this purpose, a bibliographic review was carried out between November 2017 and April 2018 in the PubMed, Academic Google, Capes Periodicals, LILACS and SCIELO databases for the last 10 years, by randomized clinical trials, bibliographic reviews and meta-analyzes of random effects, in addition to official manuals of the Ministry of Health. The selection of eligible articles and the evaluation of their methodological quality were done independently by two reviewers, and the keywords used were: 'physiotherapy'; 'humanized birth'; 'Labor pain'; and 'labor', and their correlates in the English language: 'physical therapy'; 'Humanized labor'; 'Labor pain'; and 'labor'. The physiotherapeutic strategies used during labor and humanized labor for pain relief were from Western to Eastern techniques, such as Swiss bal positioning and walking, respiratory perception, relaxation, TENS, massage, hot baths, cryotherapy, reflexology, auriculotherapy, acupuncture and acupressure.

Keywords: Physiotherapy; Humanized birth; Labor pain; Labor.

Topic: Fisioterapia

Reviewed anonymously in the process of blind peer
Received: $\mathbf{1 1 / 0 8 / 2 0 1 9}$

Approved: 21/08/2019
Sarah Arrais Ramos (iD

Faculdade Guaraí, Brasil

http://lattes.cnpq.br/3557990875511944

http://orcid.org/0000-0003-1603-0223

vanle.morais@gmail.com

Vanessa Leite de Morais

Faculdade Guaraí, Brasil

http://lattes.cnpq.br/0326151923597766

vanle.morais@gmail.com

Daniela Maristane Vieira Lopes Maciel (iD

Faculdade Guaraí, Brasil

http://lattes.cnpq.br/0591590391105455

http://orcid.org/0000-0001-5934-0219

daniela.marciel@iescfag.edu.br

\section{Referencing this:}

RAMOS, S. A.; MORAIS, V. L.; MACIEL, D. M. V. L.. Estratégias fisioterapêuticas para alívio da dor durante trabalho de parto. Scire Salutis, v.8, n.2, p.76-87, 2018. DOI:

http://doi.org/10.6008/CBPC2236-9600.2018.002.0008 


\section{INTRODUÇÃO}

Para a Organização Mundial de Saúde (OMS), o Brasil é o país com maior índice de parto cesáreo no mundo, cerca de 55,5\% (BRASIL, 2017), visto que o índice ideal é de 10 a 15\% (OMS, 2016). Esse alto índice está relacionado ao medo da dor do parto, a preocupação com a integridade da vagina e do períneo e também por preferência dos médicos por sua praticidade e conveniência, pois, é uma intervenção programada ao contrário do parto vaginal (BRASIL, 2017).

A partir da década de 80 , surgiu um movimento que tinha como uma de suas prioridades promover avanços na qualidade da assistência prestada à parturiente, reduzindo o uso de avanços tecnológicos que poderiam comprometer a saúde da parturiente e do recém-nascido, este movimento ficou conhecido no Brasil, como parto humanizado (CANESIN et al., 2010; LONGO et al., 2010).

A OMS preconiza o parto humanizado para garantir saúde ao binômio mãe-filho, reduzir as taxas de mortalidades materna e neonatal e restringir o uso de recursos tecnológicos. O Ministério da Saúde, através da Portaria no 1.459, de 24 de junho de 2011, instituiu no âmbito do Sistema Único de Saúde, a Rede Cegonha, para assegurar à mulher o direito à atenção humanizada na gravidez, parto e puerpério, através de boas práticas baseadas em evidências científicas, biossegurança e respeito aos valores culturais (BRASIL, 2014). O parto humanizado visa promover melhor assistência a parturiente, devolvendo-a o protagonismo do parto e garantindo bem estar e participação ativa da mulher no trabalho pode levar à diminuição da taxa de cesariana e duração do trabalho sem efeitos deletérios para a mãe e o feto (HANJANI et al., 2014).

A equipe multidisciplinar deve estar preparada para acompanhar todo o processo de trabalho de parto e parto, de maneira mais natural possível, orientando e respeitando os desejos e prioridades da mulher, oportunizando meios de alívio de dor não-farmacológicos (BRASIL, 2017). O parto é um evento biológico natural e apresenta-se como uma experiência de impacto emocional significativo, ela encontra-se como o pivô central nesse acontecimento, cabendo a ela controlar, interagir e protagonizar as decisões referentes ao trabalho de parto e parto, salvo nas intercorrências (ABREU et al., 2010; CASTRO et al., 2012).

O trabalho de parto é marcado por alterações hormonais e mecânicas que desencadeiam contrações uterinas regulares, dilatação do colo uterino e descida do feto pelo canal vaginal. Durante a fase de dilatação, a dor é descrita como subjetiva, aguda, visceral e difusa. Já na fase de descida fetal, a dor é somática, mais intensa e contínua, podendo sofrer interferências de fatores emocionais e ambientais (GALLO et al., 2011).

E mesmo se tratando de um evento natural, é temido pelas mulheres em função dessa dor que o acompanha; é uma experiência subjetiva e complexa que varia de mulher para mulher (MAFETONI et al., 2014). A dor é um sinal clínico do início do trabalho de parto (MAKVANDI et al., 2016); no entanto, algumas mulheres consideram que é a pior dor sentida e, muitas vezes, superior ao que esperavam (GAYESKI et al., 2010).

A dor e a ansiedade geram consequências deletérias, elevando a liberação de neurotransmissores como catecolaminas e hormônio cortisol, ambos produzidos pelas glândulas suprarrenais, que tem o papel de causar repercussões hemodinâmicas, tais quais, aumento do débito cardíaco, da pressão arterial sistêmica e da resistência vascular periférica. O débito cardíaco materno eleva entre 10 e 15\% na fase de dilatação 
cervical, sobe para $50 \%$ no período expulsivo e pode chegar a valores máximos em torno de $80 \%$ logo após o parto (SANTANA et al. 2013).

Esse fluxo sanguíneo é desviado do útero para circulação central durante as contrações uterinas, e consequentemente, observa-se o comprometimento da perfusão fetal, acidose, hipóxia fetal aumento do débito cardíaco materno (SANTANA et al., 2013; CALIK et al., 2014). As catecolaminas aumentam a contratilidade uterina contribuindo para os distorcias (SANTANA et al., 2013). E o aumento dos níveis sanguíneos de hormônios, como epinefrina, intensificará ainda mais a dor do trabalho de parto, e potencialmente prolongará a primeira e a segunda fase desse processo, resultando em uma experiência muito desagradável de parto (DOLATIAN et al., 2011; SANTANA et al., 2016).

Por esta razão que a dor é um dos principais obstáculos durante o trabalho de parto (OLIVEIRA et al., 2014), e para minimizar os efeitos deletérios, deve ser amenizada e vista positivamente, pela parturiente e membros da família, através da orientação e estratégias de alivio da dor ofertada pelo profissional fisioterapeuta durante todo o processo de trabalho de parto, proporcionando analgesia, conforto, segurança e relaxamento (ABREU et al., 2010; MAFETONI et al., 2016).

O fisioterapeuta é o profissional apto no uso de técnicas não farmacológicas para a promoção de analgesia, além de proporcionar melhorias no suporte emocional e físico da parturiente, auxiliando na redução da ansiedade e do medo (SILVA et al., 2015). O objetivo deste estudo é realizar uma revisão bibliográfica para identificar as principais estratégias utilizadas na assistência fisioterapêutica durante o trabalho de parto e parto humanizado para alívio da dor.

\section{METODOLOGIA}

O presente estudo trata-se de uma pesquisa descritiva de cunho bibliográfico, realizada nos últimos 10 anos, envolvendo publicações sobre o tema 'assistência fisioterapêutica no parto humanizado'. As palavras-chave empregadas para a busca dos materiais segundo os DeCS (Descritores em Ciências da Saúde) foram: 'Fisioterapia'; 'Parto humanizado'; 'Dor de parto'; e 'Trabalho de parto'. Além desses, seus correlatos em inglês: 'Physiotherapy'; 'Humanized birth'; 'Labor pain'; e 'Labor'.

A pesquisa em periódicos estendeu-se de novembro de 2017 a março de 2018, através das bases de dados eletrônicos como Scientific Electronic Library Online (SciELO), Google Acadêmico, Coordenação de Aperfeiçoamento de Pessoal de Nível Superior (CAPES), LILACS (Literatura Latino-Americana em Ciências da Saúde), Pubmed e documentos oficiais do Ministério da Saúde.

A seleção dos artigos elegíveis e a avaliação de sua qualidade metodológica foram feitas independentemente por dois revisores, buscou-se por ensaios clínicos randomizados e quase-randomizados, revisões bibliográficas, sistemáticas e meta-análises de efeitos randômicos. Foram selecionados um total de 73 artigos, onde 35 foram excluídos por não apresentarem compatibilidade com o tema ou exceder o tempo de investigação. Dos 45 artigos incluídos no estudo, 20 eram ensaios clínicos randomizados, 13 estudos de revisão bibliográfica, 03 de revisão sistemática, dois artigos de instituição, 01 meta-análise, 05 artigos de abordagem qualitativa, 01 estudo de caso. 


\section{DISCUSSÃO TEÓRICA}

\section{Assistência Fisioterapêutica no Trabalho de Parto e Parto}

Durante o trabalho de parto, o corpo reage a uma cascata de sentimentos e eventos fisiológicos, psicológicos, étnicos, comportamentais e culturais que se associam e interagem com os estímulos advindos do meio ambiente a qual a parturiente está inserida (SANTANA et al., 2013). O acompanhamento do fisioterapeuta no parto humanizado de baixo risco é de grande importância para garantir assistência humanizada ao parto, promovendo a valorização da gestante, utilizando seu próprio corpo, promovendo relaxamento da musculatura vaginal, controle respiratório, adequação da postura, alivio de dor na região lombrossacra (BAVARESCO et al., 2011).

Os métodos de alívio de dor são: farmacológico e não-farmacológico. Os farmacológicos apresentam limitações, pois as drogas usadas para causar analgesia no trabalho atravessa a barreira placentária e podem causar efeitos deletérios sobre a mãe e o feto Os métodos não farmacológicos visam aumentar a tolerância à dor, não possuem efeitos colaterais e são recomendados pela OMS no atendimento ao parto normal. Três princípios que são essenciais para aliviar a dor na obstetrícia incluem segurança, simplicidade e manutenção da homeostase fetal, e os métodos não farmacológicos, essas premissas (DAVIM et al., 2009; DOLATIAN et al., 2011).

As estratégias de assistência fisioterapêutica utilizadas podem ser estímulo à deambulação, adoção de posturas verticais, exercícios respiratórios, exercícios na bola, técnicas de relaxamento, analgesia por meio da Eletroestimulação Transcutânea (TENS), massagens, banhos quentes, crioterapia, acupuntura, dentre outras (SILVA et al., 2016).

\section{Posicionamento e Deambulação}

O processo de deambular é importante durante o trabalho de parto, pois proporciona a descida do feto, aumenta a frequência das contrações uterinas e acelera a dilatação cervical (GALLO et al., 2011; CASTRO et al., 2012). A redução da duração do trabalho de parto em parturientes que deambularam ou adotaram posições verticais versus posições reclinadas, foi avaliada na revisão sistemática de LAWRENCE et al., 2011, através de ensaios clínicos randomizados e quase randomizados, numa amostra total de 5.218 mulheres, recomendando que as parturientes sejam encorajadas a deambulação e a ter liberdade de posição na fase latente $(3-4 \mathrm{~cm})$ de trabalho de parto.

Por isso, a gestante deve ter liberdade para escolher a posição mais confortável (WEI et al., 2011). As posturas mais utilizadas durante o processo de trabalho de parto e parto são: a inclinação do corpo para frente com os braços sobreposto na parede, posturas eretas, posição de gato, sentada na cama utilizando almofadas, decúbito lateral esquerdo que promove o aumento da circulação útero placentária, o que provoca o aumento a produção de oxitocina, reduzindo desta forma a duração do trabalho de parto (MAZZALI et al., 2008). 


\section{Bola Suíça}

A bola suíça é um recurso bem aceito pelas gestantes durante o trabalho de parto, contribuindo para sua participação ativa, além de auxiliar no relaxamento perineal, proporcionar correção postural, favorecer a descida do feto e fortalecer os músculo levantador do ânus e pubo coccígeo (OLIVEIRA et al., 2014). O uso da bola e do cavalinho estimulam a posição vertical, permite liberdade na adoção de diferentes posições, possibilita o exercício do balanço pélvico, tem característica de objeto lúdica e é de baixo custo financeiro (OREANO et al., 2014). Auxilia, também, a mulher, a ter maior conforto e a permanecer em posições mais adequadas durante o processo do nascimento (SANTANA et al., 2016).

De acordo com um estudo de intervenção randomizado e cego realizado por Barbieri et al. (2013), 15 parturientes de baixo risco foram recrutadas em 3 grupos isômeros. 0 1으 grupo recebeu banho de aspersão com água quente à temperatura de $37^{\circ} \mathrm{C}$, por 30 minutos na região lombossacra, com a liberdade de posição; ao $2^{\circ}$ grupo, foi ofertado exercício perineal com bola suíça com pernas fletidas a $90^{\circ} \mathrm{C}$, durante 30 minutos; e o 3o grupo recebeu ambas as intervenções. Os resultados apresentados foram satisfatórios para a utilização dos recursos associados, com diminuição significativa dos escores de dor em relação aos grupos 1 e 2 quando analisados separadamente.

Uma intervenção randomizada foi realizada por Gallo et al., em 2014, para avaliação recursos não farmacológicos, duração do trabalho de parto, velocidade e descida fetal em 40 parturientes com dinâmica uterina, 4-5cm de dilatação cervical. Foi instituído um Grupo Bola (GB) que realizou exercícios de mobilidade pélvica na bola suíça, exercícios ativos de anteversão e retroversão pélvica, lateralizarão, circundarão e propulsão e um grupo controle $(\mathrm{GC})$ que realizou procedimentos da maternidade e a liberação de posição. $O$ alívio da dor foi obtido nos dois grupos e quanto ao tempo de duração do trabalho de parto não houve diferença significativa, sendo a bola suíça considerada um recurso positivo para alivio da dor no parto.

\section{Percepção Respiratória}

Os exercícios respiratórios durante o trabalho de parto têm o papel de reduzir a sensação dolorosa, otimizar os níveis de saturação materno-fetal, reduzir os níveis de tensão e garantir um melhor relaxamento (GALLO et al., 2011). Uma das técnicas indicadas durante o processo de trabalho de parto é a respiração abdominal, onde a parturiente distende o abdômen na inspiração e contrai o abdômen na expiração, através de movimentos expiratórios lentos, ao mesmo tempo em que realizam um freio labial lento e progressivo, executando uma pressão extra no abdômen contribuindo para exercer uma força adicional empurrando o feto. No período expulsivo, a parturiente respira fundo e realiza uma apneia fazendo força para expulsar o bebê relaxando a musculatura perineal.

Foram realizado quatro estudos com 19 mulheres referentes ao uso de exercícios respiratórios no trabalho de parto, constatando que 3 desses artigos demonstraram que esse método promove relaxamento nos intervalos entre as contrações, sendo que $47,4 \%$ das mulheres relataram analgesia e $53,9 \%$ relatam sentir-se relaxadas e tranquilas (MAFETONI et al., 2014). 


\section{Relaxamento}

A técnica de relaxamento utiliza exercícios mentais que trazem o benefício da diminuição do tônus muscular causando analgesia e induzindo ao aumento dos níveis de ocitocina circulante. A gestante pode posicionar-se confortavelmente numa cadeira ou deitar-se em decúbito lateral esquerdo, pois esta posição possibilita o aumento da circulação placentária, aumento do fluxo de oxitocina, redução da duração do trabalho de parto (MAZZALI et al., 2008).

O ambiente silencioso promove a sensação de conforto e relaxamento durante o parto, sendo necessário e de grande importância para que os fenômenos fisiológicos envolvidos no processo da parturição ocorram de forma adequada, como a liberação de ocitocina e endorfinas endógenas, facilitando o curso normal do trabalho de parto (GUIDA et al., 2013).

\section{Eletroestimulação Transcutânea (TENS)}

A dor durante o primeiro estágio do trabalho de parto está relacionada aos estímulos nociceptivos transmitidos pelas fibras A-delta e C das estruturas pélvicas de origem tanto visceral quanto somática, relacionadas à dilatação do colo do útero, vagina e músculos do períneo. À medida que o trabalho de parto avança os impulsos dolorosos, são transmitidos desde a raiz nervosa de T10 até S4, quando a dor se torna mais intensa e mais difusa, o que justifica sua progressão, formando uma curva ascendente à medida que se aproxima do período expulsivo (BARBIERI et al., 2013).

A TENS é utilizada para trazer alívio da dor para a gestante durante o trabalho de parto, estudos apontam sua maior efetividade é no primeiro e segundo estágios (ABREU et al., 2010; MELO et al., 2011; SANTANA et al., 2016; SHAHOEl et al., 2017), dispondo os eletrodos em áreas da medula espinhal correspondentes à T10-L1 e S2-S4, respectivamente, que são os locais de entrada dos aferentes nociceptivos. No primeiro estágio do trabalho de parto a dor é visceral, e mediada principalmente pelo sistema nervoso simpático, o qual inerva o útero, conduzindo estímulos com aferências no sistema nervoso central ao nível de T10-L1. No segundo estágio ela é somática, resultante da distensão do períneo; o qual é inervado pelo nervo pudendo, que entra pelos segmentos espinhais S2-S4 (ABREU et al., 2010).

O ensaio clínico randomizado cego de Santana et al. (2016) avaliou primíparas com 4cm de dilatação cervical no uso de TENS com largura de pulso $100 \mathrm{~ms}$ e frequência $100 \mathrm{~Hz}$, durante 30 minutos; o resultado confirmou a eficácia na aplicação de TENS no alívio de dor, no entanto, não houve alteração quanto a localização ou distribuição da dor, além de não ter sido evidenciado repercussões negativas maternoneonatais.

Na revisão sistemática de Dowswell et al. (2009), com 1466 mulheres distribuídas em 17 ensaios clínicos, treze estudos aplicaram TENS nas costas, dois para pontos de acupuntura e dois para o crânio. Considerou que há apenas evidências limitadas de que o TENS reduz a dor no trabalho de parto e não parece ter impacto (positivo ou negativo) em outros desfechos para mães ou bebês. 


\section{Massagem}

A massagem é um recurso de estimulação sensorial caracterizado pelo toque sistêmico e pela manipulação dos tecidos. No trabalho de parto, a massagem tem o potencial de promover alívio de dor, proporcionar contato físico com a parturiente, potencializando o efeito de relaxamento, diminuindo o estresse emocional e melhorando o fluxo sanguíneo e a oxigenação dos tecidos (GALLO et al., 2011).

Durante o processo de parto as parturientes relatam dor nas regiões lombrossacra e membros inferiores. A massagem se apresenta como um recurso analgésico através do relaxamento e diminuição das tensões musculares. A técnica consiste em aplicada toques leves, effeurage e fricção (MAZZALI et al., 2008). A massoterapia utiliza o deslizamento superficial com o auxílio de cremes e óleos no qual a parturiente deve se manter na posição sentada ou deitada em decúbito lateral esquerdo (CASTRO et al., 2012).

A massagem auxilia no alivio de dor, nos desconfortos reduzindo o estresse, ansiedade, promovendo o relaxamento muscular, reduzindo a fadiga, promovendo efeito sedativo, aumento da consciência corpórea, melhora do equilíbrio entre o sistema simpático e parassimpático. Apesar do pouco consentimento do uso desta técnica durante o trabalho de parto, as gestantes afirmam que esta técnica promove tranquilidade, analgesia, redução do quadro de ansiedade (BAVARESCO et al., 2011).

Pode ser realizada em todas as áreas do corpo de acordo com o consentimento da parturiente, existe várias formas de se aplicar a massagem dentre elas encontram-se: massagem do tecido conjuntivo nas zonas reflexas do baixo ventre e na região sacral, massagem com as duas mãos sobre as articulações sacro ilíacas no sentido longitudinal do occipital até o cóccix, massagem através de batidas leves com os dedos no baixo ventre de um lado para o outro, massagem leve e suave realizada com as mãos abertas de um lado ao outro na região do baixo ventre, deslizamento da região sacrococcígea até as cristas ilíacas e massagem profunda sobre a região sacral , paralelamente à coluna vertebral (BAVARESCO et al., 2011).

\section{Banhos Quentes}

Consiste em uma técnica que utiliza o chuveiro ou uma banheira com água aquecida a $37-39^{\circ} \mathrm{C}$, que apesar de poucas evidências científicas, apresentam relação com a modulação da dor durante trabalho de parto. Seu mecanismo de ação está relacionado ao sistema cardiovascular, uma vez que induz a vasodilatação periférica, redistribuindo o fluxo sanguíneo uterino. Os efeitos para o sistema musculoesquelético são relaxamento e maior elasticidade do canal vaginal, efeito ansiolítico com diminuição da liberação das catecolaminas e aumento das endorfinas (GALLO et al., 2011; SANTANA et al., 2013).

Avaliaram a intervenção do banho de chuveiro com duração de 30 minutos, à temperatura entre 36 a 38, $4^{\circ} \mathrm{C}$, em 6 primigestas à termo entre 4 e $7 \mathrm{~cm}$ de dilatação, no estudo de MAZONI, FARIA E MANFREDO, em 2009. Os resultados são sugestivos que o banho quente acelera o trabalho de parto e contribuem para o alívio de dor.

O ensaio clínico de intervenção realizado por Santana et al. (2013) incluiu 34 primigestas à termo, na fase ativa do trabalho de parto, com $4-5 \mathrm{~cm}$ de dilatação, com início dos sinais clínicos espontâneo, sem 
suporte medicamentoso e ausência de fatores de risco. O banho de chuveiro era ofertado por $30 \mathrm{~min}$, com temperatura controlada entre $37-39^{\circ} \mathrm{C}$. O resultado foi significativo no alívio da dor durante trabalho de parto. Corroborando com esse resultado o ensaio clínico controlado e randomizado de GALLO et al., em 2013, realizado com 108 gestantes que realizaram banho de imersão por 30 minutos, sugeriu redução do tempo das contrações, analgesia e conforto.

\section{Crioterapia}

É uma técnica que promove analgesia, reduzindo os estímulos dolorosos na via nervosa aferente nociceptiva e por conseguinte, redução do metabolismo e vasoconstrição periférica. Podem ser utilizada compressas frias ou bolsas de gel congelado (MAZONI et al., 2009). A crioterapia no ponto de Hoku associada à musicoterapia foi recomendada pelo estudo prospectivo, randomizado e controlado conduzido em Dehcheshmeh et al. (2015), avaliando 90 primigestas no início da fase ativa, dilatação cervical de 4cm, e antes e após cada intervenção nas dilatações 4,6 e $8 \mathrm{~cm}$.

O gelo é uma das formas mais antigas para promoção de analgesia, pois propicia a redução da via nervosa aferente nociceptiva por redução metabólica e isquêmica da vasa nervorum e nervi nervorum, devido à vasoconstrição, as parturientes podem utilizar compressas frias ou gel congelado para promovendo a redução da temperatura e a dor (BAVARESCO et al., 2009).

Foi realizado um estudo no Hospital Maternidade Carmela Dutra, no Rio de Janeiro, utilizando o gelo como recurso para a promoção de analgesia durante o trabalho de parto, sendo verificado melhorias no parto e no perfil biofísico do recém-nascido. Esse estudo concluiu que o uso da crioterapia promove redução do quadro álgico, relaxamento geral das gestantes sem produzir alterações o efeitos indesejáveis para a gestante e para feto (BAVARESCO et al., 2009).

\section{Reflexologia}

Reflexologia é método oriental não invasivo de alívio da dor. Baseia-se em um sistema de zonas e áreas reflexas nas mãos e nos pés que refletem uma imagem de todo o corpo exatamente mesma ordem e posição que no corpo. Embora existam diferentes interpretações quanto ao mecanismo de ação da Reflexologia, algumas teorias foram propostas, tais quais: teoria do controle de portão da dor e a teoria do impulso neural, que aumenta a secreção de endorfinas e encefálicas que auxiliam no alivio a dor, melhora o fluxo sanguíneo e linfático, contribuindo para eliminação de toxinas do corpo (DOLATIAN et al., 2011). Durante trabalho de parto a estimulação reflexa da glândula pituitária, hipotálamo, plexo solar e útero, podem proporcionar analgesia, principalmente na região lombar, e diminuir os níveis de ansiedade e estresse (HANJANI et al., 2014).

O ensaio clínico de Dolatian et al. (2011), com 120 primíparas em trabalho de parto ativo, $4-5 \mathrm{~cm}$ dilatação. 0 método aplicado foi massagem suave nos pés, 20 minutos em cada pé, seguida de pressão nos pontos: glândula pituitária, no centro do polegar; Plexo solar, quase quatro dedos largura abaixo da base dos dedos dos pés, localizado no centro (meio do diafragma); lombar e coluna sacral (região da medula espinhal) 
e Genital área abaixo do tornozelo. Durante a dilatação de $6-7 \mathrm{~cm}$, bem como $8-10 \mathrm{~cm}$, a intensidade da dor foi significativamente menor na reflexologia do que no grupo controle. Os resultados apontam para a possibilidade de usar reflexologia no manejo da dor.

Estudo de intervenção randomizado foi realizado com 80 primigestas, de $3-4 \mathrm{~cm}$ de dilatação por Hanjani et al. (2014), sendo submetidas a 40 minutos de reflexologia com óleo de girassol, ou seja, 20 minutos em cada pé, nos seguintes pontos glândula pituitária, hipotálamo, plexo solar e útero. O resultado sugere melhora do humor, redução da ansiedade e diminuição da intensidade da dor durante trabalho de parto.

Para Valiane et al. (2010), os resultados sugerem que a reflexologia pode diminuir a dor na primeira fase do trabalho de parto, mas sem evidencia que poderia encurtá-lo, fato observado no estudo de intervenção com 88 primigestas, com dilatação de $3-5 \mathrm{~cm}$, onde aplicaram a reflexologia por 30 minutos em cada pé, nos seguintes pontos plexo solar, áreas relacionadas com as vísceras digestivas, área pélvica, pituitária, sinusites, extremidades superiores e inferiores (lados externos dos pés), medula espinhal (lados internos dos pés), pulmões, área do peito raso, os lados nos pés, ovários, útero e trompas de falópio; e a reflexologia específica, incluindo as áreas relacionadas à dor do trabalho, como fígado, baço, rim, pituitária, plexo solar e útero.

\section{Auriculoterapia}

O ensaio clínico randomizado de Mafetoni et al. (2016), avaliou 30 gestantes em trabalho de parto espontâneo sem intercorrência, entre 4 e $7 \mathrm{~cm}$ de dilatação, aplicando técnica de auriculoterapia baseado no mapa chinês, com o objetivo de alivio da dor e redução do tempo de trabalho de parto. Foram utilizados 4 pontos: shen men, útero, área de neurastenia e endócrino, e o resultado não apresentou diferenças significativas nos escores de dor e duração do trabalho de parto diferente daquelas dos grupos placebo e controle.

\section{Acupressão}

É um dos recursos não-farmacológicos de origem oriental que auxiliam na diminuição da dor e na redução do tempo do trabalho de parto, pois agem aumentando a efetividade das contrações uterinas e estimula a produção endógena de ocitocina pela hipófise (CALIK et al., 2014; MAKVANDI et al., 2016). Além disso, a acupressão impede a elevação dos níveis de catecolamina, beta endorfinas, ACTH e cortisol na gestante, reduzindo a resposta neuroendócrina à dor. Com isso há redução dos efeitos deletérios cardiovasculares e a evolução do trabalho de parto é acelerada.

Acupressão significa pressão em pontos de acupuntura com as pontas dos dedos, sem o uso de agulhas (SEHHATIE-SHAFAIE et al., 2013; CALIK et al., 2014). Os resultados sugerem que a acupressão pode reduzir a duração do trabalho, particularmente na primeira etapa e os pontos mais estudados são Sanyinjiao / Baço 6 (SP6) e Hegu / Intestino Grosso 4 (LI4) (MOLLART et al., 2015).

Avaliando o efeito do ponto SP6 da acupuntura, que representa o ponto do fígado, baço e meridianos de rim, localizado $3 \mathrm{~cm}$ acima do ápice do maléolo medial, posterior à margem da tíbia. 0 ensaio clínico 
randomizado de CALIK et al. (2014) com 100 parturientes com 4cm de dilatação foi efetivo no controle dor no trabalho e redução da duração do trabalho.

Outro estudo corrobora com esse resultado, Hamidzadeh et al. (2012), realizaram um ensaio clínico randomizado com 100 parturientes em início de trabalho de parto espontâneo $2-3 \mathrm{~cm}$ de dilatação, estimulando o ponto LI4 (intestino) por 20min. O LI4 fica entre o primeiro e segundo ossos do metacarpo, ao lado do base do segundo metacarpiano. Hamidzadeh et al. (2012) obteve diferenças significativas em relação ao grupo controle no que tange ao alívio da dor e encurtamento do tempo de trabalho de parto, sem surgimento de reações adversas.

O estudo controlado randomizado de Ozgoli et al. (2016) comparou o efeito do ponto LI4 e BL32 (localizado no segundo forame sacral) na acupressão em 105 primigestas, em três dilatações cervicais 4-5, 67 e 8-10cm antes e após intervenção. A redução da dor foi significativamente maior nos grupos LI4 e BL32 em comparação com o controle em todos os períodos de estudo. Além disso, a acupressão no ponto BL32 foi superior ao ponto LI4 no alívio da dor na primeira e segunda, mas não na terceira intervenção.

A associação do ponto SP6 e LI4 para promover analgesia durante o trabalho de parto de primigestas, foi avaliada por SEHHATIE-SHAFAIE et al. (2013), através de um estudo controlado e randomizado com 38 gestantes em cada grupo, em trabalho de parto de início espontâneo, em torno de $4 \mathrm{~cm}$ de dilatação. A avaliação foi realizada com 4, 6, 8 e 10 dilatações antes e depois da intervenção em ambos os grupos, considerando pressão dos pontos durantes as contrações uterinas. Os resultados sugerem que a intensidade da dor teve uma diminuição significativa, imediatamente após a intervenção no grupo experimental em comparação com o grupo de controle.

\section{CONSIDERAÇÕES FINAIS}

Esta revisão permitiu dar visibilidade às produções brasileiras sobre as estratégicas fisioterapeuta para alivio da dor, o suporte físico e emocional provocado pelo fisioterapeuta durante o trabalho de parto e o parto de baixo risco parecem contribuir para sua humanização e a do nascimento ao proporcionar à parturiente bem-estar físico, redução das percepções dolorosas, aumento da confiança, redução do medo e da ansiedade, e maior consciência do processo parturitivo.

Além disso, a assistência fisioterapêutica ajuda ainda a tornar o processo de parturição mais ativo, natural e satisfatório, favorecendo uma vivência positiva na vida social e familiar da parturiente, como mulher e mãe. Acreditamos que o fisioterapeuta é o profissional da saúde que dispõe de todo o conhecimento para fornecer este suporte de forma eficiente e segura, priorizando métodos nãofarmacológicos de alívio da dor no trabalho de parto e no parto. Todavia, a presença do fisioterapeuta no auxílio à parturiente ainda é desconhecida pela maioria da população e dos profissionais de saúde, necessitando-se cada vez mais de estudos que demonstrem o seu trabalho e sua capacitação diante da maternidade e do nascimento. 


\section{REFERÊNCIAS}

ABREU, E. A.; SANTOS, J. D. M.; VENTURA, P. L.. Efetividade da eletroestimulação nervosa transcutânea no alívio da dor durante o trabalho de parto: um ensaio clínico controlado. Revista da Sociedade Brasileira para o Estudo da Dor, São Paulo, n.11, n.4, p.313-318, 2010.

BARBIERI, M.; HENRIQUE, A. J.; CHORS, F. M.; MAIA, N. L.; GABRIELLONI, M. C.. Banho quente de aspersão, exercícios perineais com bola suíça e dor no trabalho de parto. Acta Paulista Enfermagem, São Paulo, v.26, n.5, p.478-484, 2013.

BAVARESCO, G. Z.; SOUZA, R. S. O.; ALMEICA, B.; SABATINO, J. H.; DIAS, M.. O Fisioterapeuta como Profissional de Suporte à Parturiente. Ciência \& Saúde Coletiva, Rio de Janeiro, v.16, n.7, p.3259- 3266, 2011.

BRASIL. Ministério da Saúde. Cadernos Humaniza SUS: humanização do parto e do nascimento. Brasília: MS, 2014.

BRASIL. Ministério da Saúde. Diretrizes Nacionais de Assistência ao Parto Normal. Brasília: MS, 2017.

CALIK, Y. K.; KOMURCU, N.. Effects of SP6 acupuncture point stimulation on labor pain and duration of labor. Iranian Red Crescent Medical Journal, Dubai, v.16, n.10, p.164-61, 2014. DOI: http://doi.org/10.5812/ircmj.16461

CANESIN, K. F.; AMARAL, W. N.. Atuação fisioterapêutica para diminuição do tempo do trabalho de parto: revisão de literatura. FEMINA, São Paulo, v.38, n.8, p.329-333, 2010.

CASTRO, A. S.; CASTRO, A. C.; MENDONÇA, A. C.. Abordagem fisioterapêutica no pré-parto: proposta de protocolo e avaliação da dor. Fisioterapia e Pesquisa, São Paulo, v.19, n.3, p.210-214, 2012.

DAVIM, R. M. B.; TORRES, G. V.; DANTAS, J. C.. Revista da Escola de Enfermagem da USP, São Paulo, v.43, n.2, p.438445, 2009.

DEHCHESHMEH, F. S.; RAFIEI ${ }_{2}$ H.. Complementary and alternative therapies to relieve labor pain: a comparative study between music therapy and Hoku point ice massage. Complementary Therapies in Clinical Practice, Nova York, v.21, n.4, p.229-32, 2015.

DOLATIAN, M.; HASANPOUR, A.; MONTAZERI, S. H.; HESHMAT, R.; MAJD, H. A.. O efeito da reflexologia na intensidade da dor e durante o parto em primíparas. Iranian Red Crescent Medical Journal, Dubai, v.13, n.7, p.475-479, 2011.

DOWSWELL, T.; BEDWELL, C.; LAVENDER, T.; NEILSON, J. P.. Transcutaneous electrical nerve stimulation (TENS) for pain management in labour. Cochrane Database System Review, Chichester, v.15, n.2, p.1-50, 2009.

GALLO, R. B. S.; SANTANA, L. S.; MARCOLIN, A. C.; FERREIRA, C. H. J.; DUARTE, G.; QUINTANA, S. M.. Recursos nãofarmacológicos no trabalho de parto: protocolo assistencial. Femina, São Paulo, v.39, n.1, p.41-8, 2011.

GALLO, R. B. S.; SANTANA, L. S.; MARCOLIN, A. C.; QUINTANA, S. M.. A bola suíça no alivio da dor de primigestas na fase ativa do trabalho de parto. Revista da Sociedade Brasileira para o Estudo da Dor, São Paulo, v.15, n.4, p.253-255, 2014.

GAYESKI, M. V.; BRÜGGERMANN, O. M.. Métodos não farmacológicos para alívio da dor no trabalho de parto: uma revisão sistemática. Texto e Contexto: Enfermagem, Florianópolis, v.19, n.4, p.774-782, 2010.

GUIDA, N. F. B.; LIMA, G. P. V.; PEREIRA, A. L. F.. O ambiente de relaxamento para humanização do cuidado ao parto hospitalar. Reme, Belo Horizonte, v.17, n.3, p.524-530, 2013.

HAMIDZADEH, A.; SHAHPOURIAN, F.; ORAK, R. J.;; MONTAZERI, A. S.; KHOSRAVI, A.. Effects of LI4 acupressure on labor pain in the first stage of labor. Journal of Midwifery \& Women's Health, Silver Spring, v.57, n.2, p.133-138, 2012.

HANJANI, S. M.; TOURZANI, Z. M.; SHOGHI, M.. The Effect of foot reflexology on anxiety, pain, and outcomes of the labor in primigrávida women. Acta Medica Iranica, Tehran, v.53, n.8, p.507-511, 2015.

LAWRENCE, A.; LEWIS L.; HOFMEYR, G. J.; DOWSWELL, T.; STYLES, C.. Maternal positions and mobility during first stage labour. In: The Cochrane Library, Londres, v.9, n.10, 2013.

LONGO, C. S. M.; ANDRAUS, L. M. S.; BARBOSA, M. A.. Participação do acompanhante na humanização do parto e sua relação com a equipe de saúde. Revista Eletrônica de Enfermagem, Goiânia, v.12, n.2, p.385-391, 2010.

MABUCHI, A. S.; FUSTINONI, S. M.. O significado dado pelo profissional de saúde para o trabalho de parto e parto humanizado. Acta Paulista Enfermagem, São Paulo, v.21, n.3, p.420-426, 2008.

MAFETONI, R. R.; SHIMO, A. K. K.. Efeitos da auriculoterapia sobre a dor do trabalho de parto: ensaio clínico randomizado. Revista da Escola de Enfermagem da USP, São Paulo, v.50, n.5, p.726-733, 2016.

MAFETONI, R. R.; SHIMO, A. K. K.. Métodos não farmacológicos para alívio da dor no trabalho de parto: revisão integrativa. Revista Mineira de Enfermagem, Belo Horizonte, v.18, n.2, p.505-552, 2014.

MAKVANDI, S.; MIRZAIINAJMABADI, K.; SADEGHI, R.; MAHDAVIAN, M.; KARIMI, L.. Meta-analysis of the effect of acupressure on duration of labor and mode of delivery. Internacional Journal of Ginecology and Obstetrics, p.1-7, 2016. DOI: http://doi.org/10.1016/j.ijgo.2016.04.017

MARTINI, J. G.; BECKER, S. G.. A Acupuntura na analgesia do parto: percepções das parturientes. Revista de Enfermagem, Santa Maria, v.13, n.3, p.589-594, 2009.

MAZONI, S. R.; FARIA, D. G. S.; MANFREDO, V. A.. Hidroterapia durante o trabalho de parto: relato de uma prática segura. Arquivos de Ciências da Saúde, São José do Rio Preto, v.16, n.1, p.40-43, 2009.

MAZZALI, L.; GONÇALVES, R. N.. Análise do tratamento Fisioterapêutico na diminuição da dor durante o trabalho de 
parto normal. Ensaios e Ciência: Ciências biológicas agrárias e da saúde, São Paulo, v.12, n.1, p.8-17, 2008.

MELO, L. F. D.; NOBREGA, L. F.; LEMOS, A.. Estimulação elétrica transcutânea no alívio da dor do trabalho de parto: revisão sistemática e meta-análise. Revista Brasileira de Fisioterapia, São Carlos, v.15, n.3, p.175-184, 2011.

MOLLART, L. J.; ADAM, J.; FOUREUR, M.. Impact of acupressure on onset of labour and labour duration: a systematic review. Women and Birth, Camberra, v.28, n.3, p.199-206, 2015. DOI:

http://doi.org/10.1016/j.wombi.2015.03.007

OLIVEIRA, L. M. N.; CRUZ, A. G. C.. A Utilização da Bola Suíça na Promoção do Parto Humanizado. Revista Brasileira de Ciências da Saúde, São Caetano do Sul, v.18, n.2, p.175-180, 2014.

OMS. Organização Mundial da Saúde. Declaração da OMS sobre taxa de cesáreas. Genebra: OMS, 2016.

OREANO, J. M.; BRÜGGEMANN, O. M.; VELHO, M. B.; MONTICELLI, M.. Visão de puérperas sobre a não utilização das boas práticas na atenção ao parto. Ciência, Cuidado e Saúde, Maringá, v.13, n.1, p.128-136, 2014.

OZGOLI, G.; MOBARAKABADI, S. S.; HESHMAT, R.; MAJD, H. A.; SHEIKHAN, Z.. Effect of LI4 and BL32 acupressure on labor pain and delivery outcome in the first stage of labor in primiparous women: A randomized controlled trial. Complementary Therapies in Medicine, v.29, p.175-180, 2016. DOI: http://doi.org/10.1016/j.ctim.2016.10.009

SANTANA, L. S. ; GALLO, R. B. S.; FERREIRA, C. H. J.; QUINTANA, S. M.; MARCOLIN, A. C.. Transcutaneous electrical nerve stimulation (TENS) reduces pain and postpones the need for pharmacological analgesia during labour: a randomised trial. Journal of Physiotherapy, Camberra, v.62, p.29-34, 2016.

SANTANA, L. S.; GALLO, R. B. S.; FERREIRA, C. H. J.; QUINTANA, S. M.; MARCOLIN, A. C.. Efeito do banho de chuveiro no alívio da dor em parturientes na fase ativa do trabalho de parto. Revista da Sociedade Brasileira para o

Estudo da Dor, São Paulo, v.14, n.2, p.111-113, 2013.

SCHVARTZ, H. V.; PRATES, L. A.; POSSATI, A. B.; RESSEL, L. B. Estratégias de alivio da dor no trabalho de parto e parto: uma revisão integrativa. Journal of Nursing and Health, Pelotas, v.6, n.2, p.355-362, 2016.

SEHHATIE-SHAFAIE, KAZEMZADEH, R.; AMANI, F.; HESHMAT, R.. The Effect of Acupressure on Sanyinjiao and Hugo Points on Labor Pain in Nulliparous Women: a randomized Clinical Trial Fahimeh. Journal of Caring Sciences, Tabriz, v.2, n.2, p.123-129, 2013.

SHAHOEI, R.; SHAHGHEBI, S.; REZAEI, M.; NAQSHBANDI, S.. The effect of transcutaneous electrical nerve stimulation on the severity of labor pain among nulliparous women: a clinical trial. Complement Therapies in Clinical Practice, v.28, p.176-180, 2017. DOI:

http://doi.org/10.1016/i.ctcp.2017.05.004

SILVA, H. C. F.; LUZES, R.. Contribuição da fisioterapia no parto humanizado: revisão da literatura. Revista Discente da UNIABEU, v.3, n.6, p.25-32, 2015.

SILVA, L. M.; OLIVEIRA, S. M. J. V.; SILVA, F. M. B.; ALVARENGA, M. B.. Uso da bola suiça no trabalho de parto. Acta Paulista Enfermagem, São Paulo, v.24, n.5, p.656-662, 2011.

TRINDADE, F. C.; LIMA, G. A. R.. Assistência Humanizada para o alívio da dor do parto: revisão bibliográfica. Porto Velho: CUSL, 2017.

VALIANI, M.; SHIRAN, E.; KIANPOUR, M.; HASANPOUR, M.. Reviewing the effect of reflexology on the pain and certain features and outcomes of the labor on the primiparous women. Iranian Journal of Nursing and Midwifery Research, v.15, n.1, p.302-310, 2010.

WEI, C. Y. ; GUALDA, D. M. R.; SANTOS JUNIOR, H. P. O.. Movimentação e dieta durante o trabalho de parto: a percepção de um grupo de puérperas. Texto \& Contexto: Enfermagem, Florianópolis, v.20, n.4, p.717-725, 2011.

A CBPC - Companhia Brasileira de Produção Científica (CNPJ: 11.221.422/0001-03) detém os direitos materiais desta publicação. Os direitos referem-se à publicação do trabalho em qualquer parte do mundo, incluindo os direitos às renovações, expansões e disseminações da contribuição, bem como outros direitos subsidiários. Todos os trabalhos publicados eletronicamente poderão posteriormente ser publicados em coletâneas impressas sob coordenação da Sustenere Publishing, da Companhia Brasileira de Produção Científica e seus parceiros autorizados. Os (as) autores (as) preservam os direitos autorais, mas não têm permissão para a publicação da contribuição em outro meio, impresso ou digital, em português ou em tradução. 